УДК 539.1.2+534.29+530.145.61 ЯДЕРНА ФІЗИКА

https://doi.org/10.15407/jnpae2015.02.124

\author{
A. Ya. Dzyublik
}

Institute for Nuclear Research, National Academy of Sciences of Ukraine, Kyiv

\title{
TRANSMISSION OF GAMMA-QUANTA THROUGH VIBRATING TARGET
}

The transmission of the Mössbauer $\gamma$-quanta through a vibrating absorber is analyzed in the framework of the quantum theory. For this aim the photons are described by the Bialynicki - Birula's wave function. We calculated time dependence of the wave packets, which describe the transmitted $\gamma$-photons. It is shown that the squared modulus of their wave function determines the detection rate of $\gamma$-photons in full analogy with particles having a mass. The effect of anomalous transmission of Mössbauer radiation, caused by high-frequency periodic swings of the absorber, and the corresponding suppression of reactions is studied.

Keywords: Mössbauer effect, gamma-quantum, vibrating crystal, photon wave function, dynamical scattering theory, anomflous transmission.

\section{Introduction}

Interaction of the Mössbauer radiation with crystals subject to any alternating fields has been studied in numerous papers (see, for example, reviews [1, 2]), although there were discussed mainly kinematic effects. In other words, most papers took into account only single collisions of incident photons with atoms of a target. Such a kinematical approach is applicable only for thin crystals. The problem of multiple scattering of the Mössbauer rays inside the vibrating targets, having arbitrary thickness, has been addresssed in papers [3 - 7]. Unfortunately, these dynamical scattering theories are not completely consistent. In particular, in [3 - 5] the Mössbauer photons were treated as classical electromagnetic waves, goverened by the Maxwell equations. The quantum multiple scattering equations have been solved in $[6,7]$, but without introduction of the photon wave function.

Recently new features of Mössbauer forwardscattering spectra in vibrating crystals were analyzed in $[8-10]$. One of the most interesting results was obtained by Vagizov et al. [10]. They investigated the transmission of single $\gamma$-photon through vibrating absorber, having fixed the initial moment for decay of the excited $14.4 \mathrm{keV}$ level of the nuclei ${ }^{57} \mathrm{Fe}$ in the source of $\gamma$-quanta and by using the timedelay scheme. The measured detection rate revealed sharp deviation from simple exponential decay law, that was associated with a distortion of the transmitted photon shape. The authors indicated that modulation of the photon shape can be used for the writing down an information, important for the quantum cryptography and communication. The experiment [10] has been accompanied by the quasiclassical calculations. The photon was described by a classical electromagnetic wave $E(z, t)$, while the nuclei were treated as a quantum system. Large number of correlated photons of the laser pulse really can be described by the classical wave, but single photon is a quantum object, for which such notions as strengths of the electric field $\mathbf{E}(z, t)$ or the magnetic field $\mathbf{B}(z, t)$ have no clear physical sence. The calculations in [10] have been done by methods of the classical optics. In particular, the electromagnetic wave was assumed to generate E1 transitions in nuclei ${ }^{57} \mathrm{Fe}$, although their excited $14.4 \mathrm{keV}$ level $3 / 2^{+}$and the ground one $1 / 2^{+}$are coupled by M1 transitions.

Of course, it would be much better to describe the photon with the help of the wave function. Such photon wave function has been derived by Bialynicki - Birula [11] and Sipe [12]. Its squared modulus defines the probability density to detect the photon energy $\hbar \omega$ inside some space interval at the moment $t$. This differs the photon wave function $\mathbf{F}(\mathbf{r}, t)$ from the wave function $\Psi(\mathbf{r}, t)$ of particles with mass, which determines the probability to find just the particle in any interval.

We develop here strict quantum-mechanical description of single $\gamma$-photon transmission through vibrating absorber. The photon is described by the wave function, proposed in [11, 12]. This wave function allows to describe the photon almost in the same manner as it is done for massive particles. The present paper seems to be the first application of such wave function in the $\gamma$-optics. Moreover, special attention is paid to the effect of anomalous transmission of the Mössbauer radiation through absorbers oscillating with high frequency, first predicted in [7] and observed in [5]. While only harmonic oscillations of the absorber were taken into account in the theory [10], we regard arbitrary periodic motion of the absorber as a whole. Such approach may be useful in studies of vibrations with large amplitude, when the role of anharmonicity of vibrations becomes significant.

(C) A. Ya. Dzyublik, 2015 


\section{Scattering amplitude}

Let the absorber occupy the region $0 \leq z \leq D$, where $D$ is the thickness of the slab. We assume that $\gamma$-quanta are incident perpendicularly to the surface along the axis $z$, and all the nuclei of the absorber vibrate in unison also along the axis $z$. We consider general case of arbitrary periodic oscillations of the absorber, when the corresponding displacements of all the nuclei from their equilibrium positions are defined by a periodic function $X\left(t+t_{0}\right)=X\left(t+t_{0}+T\right)$ with the period $T=2 \pi / \Omega$, circular frequency $\Omega$, and the moment $t_{0}$, related to the initial phase of oscillations $\phi_{0}$ by $\phi_{0}=\Omega t_{0}$. The nuclear sublevels are assumed to be not split and trerefore the polarizations of photons are not mixed during passage through the target

The Raman scattering of $\gamma$-quanta in vibrating crystal leads to an appearance of waves with the frequencies $\omega_{n}=k_{n} c=\omega-n \Omega$, where $n$ is an integer. In thick targets a rescattering of photons between the states $k_{n}, \lambda$ and $k_{n^{\prime}}, \lambda$ should be taken into account. The corresponding coherent Raman scattering amplitude of $\gamma$-quanta by the nucleus to zero angle is given by $[6,7]$

$$
\begin{gathered}
f_{N}^{\left(n^{\prime}, n\right)}(0)= \\
-p\left(\frac{2 I_{e}+1}{2 I_{g}+1}\right) \frac{\Gamma_{\gamma}}{4 k_{0}} e^{-2 W(\mathbf{k})} \sum_{m=-\infty}^{\infty} \frac{a_{m-n^{\prime}}^{*} a_{m-n} e^{i\left(n^{\prime}-n\right) \phi_{0}}}{\hbar\left(\omega-\omega_{0}^{\prime}-m \Omega\right)+i \Gamma / 2},
\end{gathered}
$$

where $p$ is the relative concentration of the Mössbauer isotope, $I_{e}$ and $I_{g}$ are the nuclear spins in the excited and ground states, $e^{-2 W(\mathbf{k})}$ is the Debye - Waller factor $\left(2 W=<(\mathbf{k u})^{2}>\right.$, where $\mathbf{u}$ is the disolacement of the atom from its equilibrium position due to thermal vibrations, the brackets mean the averaging over phonons), $\Gamma$ and $\Gamma_{\gamma}$ are respectively the total and radiative widths of the resonant nuclear level with the energy $h \omega_{0}^{\prime}$.

The coefficients $a_{n}$ are determined by

$$
a_{n}=\frac{1}{T} \int_{-T / 2}^{T / 2} d t e^{-i n \Omega t} e^{i k X(t)}
$$

They are nothing but the Fourier-expansion coefficients for the exponent $e^{i k X(t)}$, i.e.,

$$
e^{i k X(t)}=\sum_{n=-\infty}^{\infty} a_{n} e^{i n \Omega t}
$$

By definition, the integral (2) for the coefficient $a_{n}$ denotes the following integral sum:

$$
a_{n}=\lim _{\Delta t \rightarrow 0} \frac{1}{T} \sum_{p=0}^{N-1} e^{-i n \Omega t} p e^{i k X\left(t_{p}\right)} \Delta t,
$$

where $\Delta t=T / N$ and $t_{p}=p \Delta t$. It may be rewritten as

$$
a_{n}=\lim _{N \rightarrow \infty} \frac{1}{N} \sum_{p=0}^{N-1} e^{-2 \pi i n(p / N)} e^{i k X\left(t_{p}\right)}
$$

Then one can write down

$$
\sum_{n=-\infty}^{\infty} a_{n} a_{n+m}^{*}=
$$

$$
\lim _{N \rightarrow \infty} \sum_{p, p^{\prime}=0}^{N-1} S_{N}\left(p, p^{\prime}\right) \exp \left\{i k\left[X\left(t_{p}\right)-X\left(t_{p^{\prime}}\right)\right]\right\} e^{-2 \pi i m(p / N)},
$$

where we introduced the following notation:

$$
S_{N}\left(p, p^{\prime}\right)=\frac{1}{N} \sum_{n=-N / 2}^{N / 2-1} e^{2 \pi i n\left(p-p^{\prime}\right) / N} .
$$

The summation gives

$$
S_{N}\left(p, p^{\prime}\right)=\frac{1}{N} e^{-i \pi N\left(p-p^{\prime}\right)} \frac{1-e^{2 \pi i\left(p-p^{\prime}\right)}}{1-e^{2 \pi i\left(p-p^{\prime}\right) / N}} .
$$

It is easily seen that for $p \neq p^{\prime}$

$$
\mathrm{S}_{\mathrm{N}}\left(\mathrm{p}, \mathrm{p}^{\prime}\right) \rightarrow 0 \quad \text { as } \quad \mathrm{N} \rightarrow \infty .
$$

For $p=p^{\prime}$ from the definition (7) one gets

$$
\lim _{N \rightarrow \infty} S_{N}(p, p)=1 .
$$

Combining (9) and (10) one writes

$$
\lim _{N \rightarrow \infty} S_{N}\left(p, p^{\prime}\right)=\delta_{p p^{\prime}}
$$

where $\delta_{m 0}$ is the Kronecker symbol. Substitution of this expression into (6) gives

$$
\sum_{n=-\infty}^{\infty} a_{n} a_{n+m}^{*}=\lim _{N \rightarrow \infty} \sum_{p=0}^{N-1} e^{2 \pi i m p / N} .
$$

Performing the summation over $\mathrm{p}$ we arrive finally at

$$
\sum_{n=-\infty}^{\infty} a_{n} a_{n+m}^{*}=\delta_{n m}
$$

The relation (13) can be called an addition 
theorem for the expansion coefficients $a_{n}$ in close analogy with the theorem for Bessel functions [13].

\section{The photon wave function}

The photon wave function has two vector components with helicities $\lambda= \pm 1[11,12]$ :

$$
\mathbf{F}_{\lambda}(\mathbf{r}, t)=\int d \mathbf{k} \sqrt{\hbar \omega} \mathbf{e}_{\lambda}(\mathbf{k}) f_{\lambda}(\mathbf{k}) \frac{e^{i \mathbf{k r}-i \omega t}}{(2 \pi)^{3 / 2}},
$$

where $\mathbf{e}_{\lambda}(\mathbf{k})$ are the polarization vectors, the frequency $\omega=k c$ and the weight function $f_{\lambda}(k)$, normalized as

$$
\sum_{\lambda= \pm 1} \int d \mathbf{k}\left|f_{\lambda}(\mathbf{k})\right|^{2}=1
$$

The modulus squared of the photon wave function $\mathbf{F}_{\lambda}(\mathbf{r}, t)$ determines a density of the photon's mean energy at a given position and time $[11,12]$.

Since the energy distribution of the Mössbauer radiation is very narrow, we can write down the initial wave packet, which describes the incident photon, as

$$
\mathbf{F}_{\lambda}^{i n}(z, t) \approx \mathbf{e}_{\lambda} \sqrt{\hbar \omega_{0}} \Psi_{i n}(z, t),
$$

where

$$
\Psi_{i n}(z, t)=\int_{-\infty}^{\infty} d \omega g_{i n}(\omega) e^{-i \omega t_{r}},
$$

$t_{r}=t-z / c$ represents the retarded time

$$
g_{i n}(\omega)=-\frac{1}{2 \pi i}\left(\frac{\Gamma_{s}}{\hbar}\right)^{1 / 2} \frac{1}{\omega-\omega_{0}+i \Gamma_{s} / 2 \hbar} .
$$

Here $\Gamma_{s}$ and $\hbar \omega_{0}$ are respectively the width and energy of the excited level of the emitting nucleus. In Eq. (16) we took into account that $\omega \approx \omega_{0}$ since the width $\Gamma_{s}<<\hbar \omega_{0}$. Factorization of the photon wave function (16) allows us to interpret the function $\overline{\left|\Psi_{i n}(z, t)\right|^{2}}$ as the probability density of finding the photon in vicinity of the plane $z=$ const at the moment $t$. For brevity, from now on we use the photon wave function in units of $\sqrt{\hbar \omega_{0}} \mathbf{e}_{\lambda}$.

The wave packet (16) reaches the target at the moment $t=0$. In experiments [10] this initial moment was fixed by detection of the $122 \mathrm{keV}$ photon, leading to the population of the $14.4 \mathrm{keV}$ level in ${ }^{57} \mathrm{Fe}$. In addition, the initial phase of vibrations $\phi_{0}=\Omega t_{0}$ was also fixed with respect to the starting moment $t=0$.
Substitution of Eq. (12) into Eq. (10) yields

$$
\Psi_{i n}(z, t)=\left(\Gamma_{s} / \hbar\right)^{1 / 2} e^{-i \omega_{0} t_{r}-\Gamma_{s} t_{r} / 2 \hbar} \theta\left(t_{r}\right),
$$

where

$$
\theta\left(t_{r}\right)= \begin{cases}1, & t_{r}>0 \\ 0, & t_{r}<0 .\end{cases}
$$

is the Heaviside step function. The function (19) is normalized as

$$
\int_{0}^{\infty} d t_{r}\left|\Psi_{i n}(z, t)\right|^{2}=1
$$

that corresponds to one photon incident on the unit square of the target.

The complete wave function, which describes the photon passing through the target, is

$$
\Psi(z, t)=\int_{-\infty}^{\infty} d \omega g_{\text {in }}(\omega) \psi_{k}(z, t),
$$

where the function $\psi_{k}(z, t)$ satisfies the multiple scattering equations, given in [14], when the incident photons are described by the plane waves $\exp (i k z-i \omega t)$.

The solutions $\psi_{k}(z, t)$ differ by the index $j$, so that the complete wave function is represented by a sum

$$
\psi_{k}(z, t)=\sum_{j=-\infty}^{\infty} \psi_{k}^{(j)}(z, t)
$$

with the partial waves $\psi_{k}^{(j)}(z, t)$ having the components with different frequencies:

$$
\psi_{k}^{(j)}(z, t)=\sum_{n=-\infty}^{\infty} C_{j}(n) e^{i K_{j} z-i \omega_{n} t},
$$

where the wave vectors $K_{j}=\left\{0,0, k+\Delta_{j}(\omega)\right\}$, and $\Delta_{j}(\omega)$ denotes the difference of the wave vectors in the medium and vacuum. Note that inside the target one can approximate all the vectors $k_{n}$ by $k$.

Multiple scattering equations for the wave function $\psi_{k}(z, t)$ far from the Bragg condition can be reduced to a set of algebraic equations

$$
\sum_{n=-\infty}^{\infty} A_{n^{\prime} n} C_{j}(n)=\Delta_{j}(\omega) C_{j}\left(n^{\prime}\right)
$$

where the matrix

$$
A_{n^{\prime} n}=(2 \pi \rho / k)\left[f_{c o h R}(0) \delta_{n n^{\prime}}+f_{c o h}^{\left(n^{\prime}, n\right)}(0)\right],
$$


$\rho$ is a density of atoms in the absorber, $f_{\text {cohR }}(0)$ is the coherent Rayleigh forward-scattering amplitude of $\gamma$-quanta by electrons of the atom in the absence of the forced vibrations. Its real part reads

$$
\operatorname{Ref} f_{\text {cohR }}(0)=-r_{0} Z
$$

where $r_{0}=e^{2} / m c^{2}$ is the classical electron radius, $e$ and $m$ are charge and mass of the electron, $Z$ is the number of atomic electrons. The amplitudes obey the following boundary condition at the face surface $(z=0)$ of the target [7]:

$$
\sum_{j=-\infty}^{\infty} C_{j}(n)=\delta_{n 0} .
$$

By substituting (1) into Eqs. (25), (26) and using the addition theorem (19) one finds the eigenvalues of the matrix $A$ (see also [7]),

$$
\Delta_{j}(\omega)=\Delta_{e}+\Delta_{N}(\omega-j \Omega),
$$

where the term associated with the electrons

$$
\Delta_{e}=(2 \pi \rho / k) f_{\text {cohR }}(0)
$$

and the nuclear part

$$
\Delta_{N}(\omega)=-\rho p \frac{\pi}{k^{2}}\left(\frac{2 I_{e}+1}{2 I_{g}+1}\right) e^{-2 W(\mathbf{k})} \frac{\Gamma_{\gamma} / 2}{\hbar\left(\omega-\omega_{0}^{\prime}\right)+i \Gamma / 2} .
$$

The corresponding eigenvectors, which satisfy the constraint (28), are

$$
C_{j}(n)=a_{j} a_{j-n}^{*} e^{i n \phi_{0}},
$$

where $a_{j}$ determines the amplitude of the $j$ th wave and $a_{j-n}^{*} e^{i n \phi_{0}}$ the fraction of the Raman component with the frequency $\omega_{n}$. Thus, such a wave $\psi_{k}^{(j)}(z, t)$ inside the target $(0 \leq z \leq D)$ may be written as

$$
\psi_{k}^{(j)}(z, t)=a_{j} e^{i \Delta_{j}(\omega) z-i \omega_{j} t_{r}} e^{-i k X\left(t+t_{0}\right)} e^{i j \phi_{0}},
$$

where we took into account the expansion (17). The transmitted wave $\psi_{k}^{(j)}(z, t)_{t r}$ behind the vibrating target $(z>D)$ is described by the same formula (33) but with the factor $e^{i \Delta_{j}(\omega) D}$ instead of $e^{i \Delta_{j}(\omega) z}$.

It is convenient to replace the energies by the dimensionless parameters

$$
x=\frac{2 \hbar\left(\omega-\omega_{0}^{\prime}\right)}{\Gamma}, x_{j}=\frac{2 \hbar\left(\omega_{0}-\omega_{0}^{\prime}-j \Omega\right)}{\Gamma} .
$$

The modulus squared of the wave function (22) is calculated with the aid of (33), yielding

$$
\left|\Psi_{t r}(\mathbf{r}, t)\right|^{2}=\left(\gamma_{s} / \tau_{N}\right) e^{-\mu_{e} D}\left|\sum_{j=-\infty}^{\infty} \frac{a_{j}}{2 \pi} \int_{-\infty}^{\infty} \frac{d x e^{-i x \tau / 2}}{x-x_{j}+i \gamma_{s}} \exp \left(-\frac{i \beta / 2}{x+i}\right) e^{i j \phi_{0}}\right|^{2}
$$

where $\gamma_{s}=\Gamma_{s} / \Gamma$, and $\mu_{e}=2 \operatorname{Im} \Delta_{e}$ is the absorption coefficient associated with the electrons, $\tau=t_{r} / \tau_{N}$ is the dimensionless time measured in units of the nuclear lifetime $\tau_{N}=\hbar / \Gamma$,

$$
\beta=\sigma_{0} e^{-2 W(\mathbf{k})} n_{0}
$$

is the dimensionless thickness parameter, depending on the resonance value of the absorption cross section

$$
\sigma_{0}=\frac{2 \pi}{k_{0}^{2}}\left(\frac{2 I_{e}+1}{2 I_{g}+1}\right) \frac{\Gamma_{\gamma}}{\Gamma}
$$

and the number of the resonant nuclei per unit absorber's surface $n_{0}=p \rho D$.

In standard experiments the initial moment of decay $t=0$ is not fixed. The corresponding flux of photons behind the absorber $\mathfrak{I}_{t r}$ is obtained by averaging (35) over time and phase:

$$
\mathfrak{I}_{t r}=\mathfrak{I}_{0} e^{-\mu_{e} D} \sum_{j=-\infty}^{\infty}\left|a_{j}\right|^{2} \frac{\gamma_{s}}{\pi} \int_{-\infty}^{\infty} \frac{d x}{\left(x-x_{j}\right)^{2}+\gamma_{s}^{2}} \exp \left(-\frac{\beta}{1+x^{2}}\right),
$$

where $\mathfrak{I}_{0}$ is the flux of incident photons.

\section{Suppression of inelastic channels and reactions}

Let us consider now the case of high-frequency vibrations, when

$$
\beta / 2 \Omega \tau_{N} \ll<1 .
$$

Moreover, we suppose that the incident radiation is tuned closely to the transition in the absorber, $\omega_{0} \approx \omega_{0}^{\prime}$. In this case the forward-scattering spectrum consists of well resolved sidebands separated by $\Omega$. Now $\Delta_{N}(\omega-j \Omega) \approx 0$ if $j \neq 0$ and, as a result, the function $\psi_{k t r}(z, t)$ takes the form

$$
\psi_{k t r}(z, t)=e^{i \Delta_{e} D} e^{-i \omega t_{r}}\left[1+a_{0}\left(e^{i \Delta_{N}(\omega) D}-1\right) e^{-i k X\left(t+t_{0}\right)}\right] .
$$


The photon wave function is obtained by using Eqs. (18) and (40) in (22). The resulting integral over $\omega$ can be evaluated with the help of the procedure proposed in [15]. Then in the simplest case of $\omega_{0}=\omega_{0}^{\prime}$ and $\gamma_{s}=1$ one has

$$
\begin{aligned}
\Psi_{t r}(z, t) & =\left\{1+a_{0} e^{-i k X\left(t+t_{0}\right)}\left[J_{0}(\sqrt{\beta \tau})-1\right]\right\} \times \\
& \times e^{i \delta} \boldsymbol{D} \frac{1}{\sqrt{\tau_{N}}} e^{-i \omega_{0} t_{r}-\tau / 2} \theta\left(t_{r}\right) .
\end{aligned}
$$

The corresponding probability density, averaged over the phase $\varphi_{0}$, will be

$$
\begin{gathered}
\overline{\left|\Psi_{t r}(z, t)\right|^{2}}=\left\{\left(1-\left|a_{0}\right|^{2}\right)+\left|a_{0}\right|^{2} J_{0}^{2}(\sqrt{\beta \tau})\right\} \times \\
\times e^{-\mu_{e} D} \frac{1}{\tau_{N}} \exp (-\tau) \theta(\tau) .
\end{gathered}
$$

Note that here the term $\left(1-\left|a_{0}\right|^{2}\right)$ is a sum of the amplitudes moduli squared for all the waves $\psi_{k}^{(j)}(z, t)$ except for one with $j=0$. So from the derived formulas one sees that the attenuation of all the waves with $j \neq 0$ is only caused by the interaction with electrons (photoeffect, etc.), while the wave $\psi_{k}^{(0)}(z, t)$ is absorbed by the vibrating in unison nuclei in the same manner as in the case of a motionless target. Such an anomalous absorption is ensured by a coherent mixing of the waves with different frequencies, which form the wave $\psi_{k}^{(j)}(z, t)$. By using the addition theorem (21) for the Fourier coefficients $a_{n}$ we find that the absorption amplitude $M_{j}$ of such $j$ th partial wave by the vibrating nucleus is

$$
M_{j} \sim \sum_{n=-\infty}^{\infty} a_{-n} a_{j-n}^{*}=\delta_{j 0} .
$$

It is interesting that although every individual wave with frequency $\omega_{n}$ interacts with the nuclei, their coherent sum $\psi_{k}^{(j \neq 0)}(z, t)$ does not. In the case of the simple sinusoidal vibrations, when the forced dispacements of atoms are described by the function $X(t)=A_{0} \sin \left(\Omega t+\varphi_{0}\right) \quad$ the coefficients $a_{n}=J_{n}\left(k A_{0}\right)$, one can achieve the complete suppression of inelastic channels and reactions when modulation index $k A_{0}$ is a root of the equation $J_{0}\left(k A_{0}\right)=0$.

Of course, the most direct way to observe such a phenomenon would be a measurement of the yield of conversion electrons ejected from the vibrating slab, whose nuclei are excited by the Mössbauer radiation. The corresponding reaction cross section at the nucleus with coordinates $\mathbf{r}$ is easily obtained by making use of the expression (43):

$$
\sigma_{r}(\mathbf{r})=\left|a_{0}\right|^{2} \sigma_{r}^{(0)}(\mathbf{r})
$$

where $\sigma_{r}^{(0)}(\mathbf{r})$ denotes the reaction cross section in the point $\mathbf{r}$ of the target at rest. From Eqs. (36), (37) it is clearly seen that only the partial wave $\psi_{k}^{(0)}(\mathbf{r}, t)$ takes part in the reaction, while the contribution of all the others with $j \neq 0$ is negligible at frequencies $\Omega \gg \beta / 2 \tau_{N}$.

\section{Conclusion}

The photon wave function $\Psi(z, t)$ in our formulas plays the same role as the electric field strength of the classical wave $E(z, t)$, used in the calculations [10]. Our Eq. (28) for $\left|\Psi_{t r}(z, t)\right|^{2}$ in the case of harmonic vibrations agree with Eq. (11), derived in Ref. [10] for $|E(z, t)|^{2}$. Therefore the present theory can be regarded as a foundation of the semi-classical approach [10].

Numerical calculations of the transmitted flux $|E(z, t)|^{2}$, performed in Ref.[10], well reproduce the experimental data. Hence, there is no need to repeat numerical calculations for the time-dependent function $\left|\Psi_{t r}(\mathbf{r}, t)\right|^{2}$ in order to get the same result. Since $\left|\Psi_{t r}(z, t)\right|^{2}$ well describes the experimental value of the count rate of $\gamma$ photons measured in [10], one can really interpret $\left|\Psi_{t r}(z, t)\right|^{2} \Delta t$ as the probability of detecting the $\gamma$ photon in the time gate from $t$ to $t+\Delta t$. For narrow wave packets such a statement is equivalent to the statement that the correspondent function $|\mathbf{F}(z, t)|^{2} \Delta t$ means the probability density of detecting the energy $\hbar \omega$ in the same time interval. Thus, one can treat the experiment [10] as the proof that the function $\mathbf{F}(\mathbf{r}, t)$ is really the photon wave function. This reveals tight binding of massless photons and particles having the mass.

The anomalous transmission of Mössbauer radiation through vibrating crystal, observed in [5], has been explained there partly by the coherent enhancement of the radiative channel and partly by the anomalously weak resonance absorption. But according to Eq. (42) the anomalously transmitting part of the Mössbauer radiation $\left(1-\left|a_{0}\right|^{2}\right)$ is only ensured by a weak absorption of the partial $\gamma$-waves $\psi_{j \neq 0}$. The remaining partial wave with $j=0$, which 
enters the $\gamma$-wave packet, has the same time dependence as the wave function of photon transmitted through a motionless absorber. This means that there is no decay acceleration. Thus, the high-frequency vibrations provide the reaction suppression, but not the radiative enhancement.
It is worth to add also that our calculations can be useful in studies of deviations of forced vibrations from the sinusoidal law caused by anharmonicity or any other reasons.

I am indebted to Dr. A. G. Magner for helpful discussion of the results.

\section{REFERENCES}

1. Kopcewicz M., Mössbauer effect studies of amorphous metals in magnetic radiofrequency fields // Struct. Chem. - 1991. - Vol. 2. - P. 313 - 342.

2. Makarov E.F., Mitin A.V. Gamma-resonance spectroscopy of solid body under high-frequency excitation // Usp. Fiz. Nauk. - 1976. - Vol. 120. - P. 55 - 84.

3. Mitin A.V. Coherent transmission of polarized $\gamma$-radiation in the field of sound wave // Sov. Quant. Electron. - 1976. - Vol. 3. - P. 840 - 843.

4. Tsankov L.T. The spectrum of Mössbauer radiation passed through a vibrating resonant medium // J. Phys. A: Math. Gen. - 1980. - Vol. 13. - P. 2959 - 2967.

5. Shvyd'ko Yu.V., Smirnov G.V., Enhanced yield into the radiative channel in Raman nuclear resonant forward scattering // J. Phys.: Condens. Matter. - 1992. Vol. 4. - P. 2663 - 2685.

6. Dzyublik A.Ya. Effect of forced vibrations on scattering of X-Rays and Mössbauer radiation by a crystal (1) // phys. stat. sol. (b). - 1984. - Vol. 123. - P. 53 - 64.

7. Dzyublik A.Ya. Effect of forced vibrations on scattering of X-Rays and Mössbauer radiation by a crystal (2), Dynamical effects // phys. stat. sol. (b). 1986. - Vol. 134. - P. 503 - 513.
8. Sadykov E.K., Yurichuk A.A. Thickness effect for Mössbauer samples excited by an alternating field // Pis'ma v ZhETF. - 2014. - Vol. 99. - P. 195 - 200. [JETP Lett. - 2014. - Vol. 99. - P. 174 - 178].

9. Vagizov F., Shakhmuratov R., Sadykov E.K. Application of the Mössbauer effect to the study of optoacoustic phenohenomena // phys. stat. sol. (b). - 2015. - Vol. 252. - P. 469 - 475.

10. Vagizov F., Antonov V., Radeonychev Y.V. et al. Coherent control of the wavefronts of recoilless $\gamma$-ray photons // Nature. - 2014. - Vol. 508. - P. 80 - 83.

11.Bialynicki-Birula $J$. On the wave function of the photon // Acta Phys. Polonica. - 1994. - Vol. 86. P. 97 - 116.

12. Sipe J.E. Photon wave function // Phys. Rev. A. 1995. - Vol. 52. - P. 1875 - 1883.

13. Gradshtein I.S., Ryzhik I.M. Tables of Integrals, Sums, Series and Products. - Moscow: Izd-vo Fiz.-Mat., 1963. - $1108 \mathrm{p}$.

14. Goldberger M.L., Watson K.M. Collision Theory. New York: Wiley, 1964. - 919 p.

15. Lynch F.J., Holland R.E., Hammermesh M. // Phys. Rev. - 1960. - Vol. 120. - P. 513.

\section{О. Я. Дзюблик}

Інститут ядерних досліджень НАН України, Київ

\section{ПРОХОДЖЕННЯ ГАММА-КВАНТІВ КРІЗЬ ВІБРУЮЧИЙ ПОГЛИНАЧ}

У рамках квантової теорії аналізується проходження мессбауерівських $\gamma$-квантів крізь вібруючий поглинач. 3 цією метою фотони описуються хвильовою функцією Бялініцкі - Бірули. Ми розрахували часову залежність хвильових пакетів, які описують $\gamma$-фотони, що пройшли. Показано, що квадрат модуля цієї хвильової функції визначає швидкість детектування $\gamma$-фотонів у повній відповідності з частинками, які мають масу. Вивчається ефект аномального проходження мессбауерівського випромінення, викликаний високочастотними періодичними коливаннями поглинача, та відповідне подавлення реакцій.

Ключові слова: ефект Мессбауера, гамма-квант, вібрації, хвильова функція фотона, динамічна теорія розсіяння, аномальне проходження.

\section{А. Я. Дзюблик \\ Институт ядерных исследований НАН Украины, Киев}

\section{ПРОХОЖДЕНИЕ ГАММА-КВАНТОВ СКВОЗЬ ВИБРИРУЮЩИЙ ПОГЛОТИТЕЛЬ}

В рамках квантовой теории анализируется прохождение мессбауэровских $\gamma$-квантов сквозь вибрирующий поглотитель. С этой целью фотоны описываются волновой функцией Бялиницки - Бирулы. Мы рассчитали временную зависимость волнового пакета, который описывает прошедшие $\gamma$-фотоны. Показано, что квадрат модуля этой волновой функции определяет скорость детектирования $\gamma$-фотонов в полном соответствии с частицами, имеющими массу. Изучается эффект аномального прохождения мессбауэровского излучения, вызванный высокочастотными периодическими колебаниями поглотителя, и соответствующее подавление реакций.

Ключевые слова: эффект Мессбауэра, гамма-квант, вибрации, волновая функция фотона, динамическая теория рассеяния, аномальное прохождение. 